\title{
Tree based Energy Balanced Routing Protocol by using Clustering and Compressive Sensing Technique
}

\author{
Smriti Salaria \\ Department of Computer Science and Engineering \\ DAV University, Jalandhar \\ Punjab, India
}

\begin{abstract}
The quick expansion in network multimedia equipments has permitted additional real-time digital services for example online games and work facility, video conferencing and distance education to develop to be the conventional internet tasks. WSN has developed into huge area of research in computational theory due to its broad range of applications. But because of restricted battery power, the energy consumption has turn into major restrictions of WSNs protocols. Though many protocols have been introduced to improve the energy efficiency further but still much enhancement can be done. GSTEB has shown relatively significant results over the existing WSNs protocols. But it has ignored the use of the three things like the effect of the mobile sink, Clustering and the effect of the compressive sensing. To defeat the constraints of the earlier work a new improved technique is proposed in this research paper. The future technique has the capability to overcome the restrictions of the GSTEB routing protocol by using the compressive sensing and level based clustering.
\end{abstract}

Keywords

Wireless Sensor Network, Cluster, GSTEB

\section{INTRODUCTION}

Wireless sensor network is a network that consist huge number of sensor nodes heavily deployed in defined area to sense the data and send the sensed data to committed node called base station. Base station collects all data from the sensor nodes and sends the relevant information to the end users. For transmit of data on the network WSN require suit of network protocols. Routing protocols are the set of rules used by the routers to decide the most suitable path and then send data through the appropriate path to the destination. There are number of routing protocols that are used in WSN for example:-LEACH, TBC, PEGASIS and GSTEB. The battery life, storage capacity, low bandwidth and resources are limited in case of WSN. Due to this battery consumption and storage constraint many problems and challenges occur in WSN.

\subsection{Architecture of WSN}

Wireless sensor network consists number of wireless sensor nodes that are heavily deploy in large area as shown in Fig 1. In which the data will be transmitted via a multi-hop base station. The base station collects all the data from the sensor nodes and sends important data to the end user. The nodes are scattered in the sensor field to collect the data required. The sink node collects the data from the neighboring sensor nodes and analyzes it and forwards it to the remote controller. The Wireless Sensor Network can have single sink node or multiple sink nodes depending on the area under analysis. The nodes can be static or mobile. After the sink node's energy depletes a new node with sufficient amount of energy is selected a sink node.

\author{
Ridhi Kapoor \\ Department of Computer Science and Engineering \\ DAV University, Jalandhar \\ Punjab, India
}

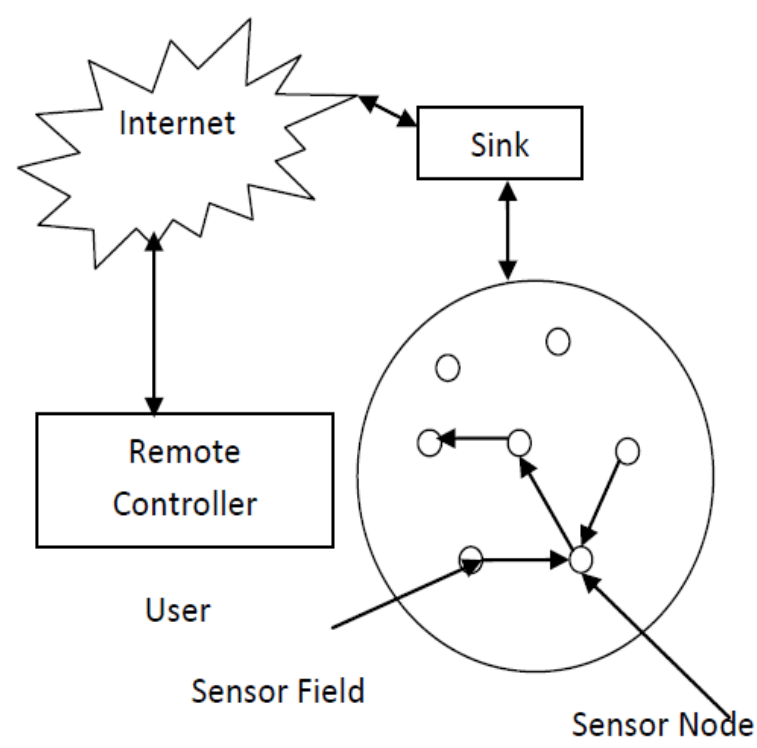

Fig 1: Architecture of WSN

\section{GSTEB}

GSTEB is a routing protocol. It is a tree based protocol. The main plan of GSTEB is to increase the network lifetime for different applications. The operation of GSTEB is divided into four phases:

- Initial phase

- Tree constructing phase

- Self-organized data collecting and transmitting phase

- Information exchange phase

In first block, BS assigns a root node and broadcast its ID and its coordinate to all sensor nodes. Then in second block, the network calculate the path by transmit the path information from BS to sensor nodes or by having the same tree structure being energetically and individually built by each node. For both cases, GSTEB can alter the root and rebuild the routing tree with short interruption and low energy consumption. In third block, after the routing tree construction, each sensor node collects information and generates a DATA_PKT which can send to BS. The TDMA time slot is also used for collecting the information from each and every node. In fourth block, the collected data is transmitted to the base station. The GSTEB protocol is compared with the other existing protocols LEACH, PEGASIS and HEED. The author showed that the performance of GSTEB is better than the others and it achieves the energy consumption. 


\section{LITERATURE SURVEY}

Han et al. (2014), [1] have discussed many protocols like PEGASIS, LEACH, HEED, TBC, PEDAP and GSTEB. A general self organized tree based energy level routing protocol has compared with other protocols. GSTEB build routing tree by using a method where, BS assigns a root node and broadcast this selection to all sensor nodes then each node starts to selects its parent nodes from its neighbor nodes, thus making GSTEB a dynamic protocol. GSTEB has shown better result than other protocols in energy balancing consumption thus it has increased the lifetime of WSNs. Hwang et al. (2014), [2] have proposed compressive sensing based WSN, that exploit channel gain to broadcast and identify signals competently. Assuming that calculated signals at each sensor are correlated and sparse at some basis domain, they suggest a novel sensor selection scheme and linked signaling channel design to improve detection performance. The simulation results show that the proposed method support reduction in the number of measurements by $60 \sim 80 \%$ for a wide range of sparsity level at high and low SNRs. Naguyen et al.(2013), [3] have discussed the cluster based energy efficient data gathering using CS that proposed to extensively decrease the energy consumption associated to data collection in such networks. Both compressive sensing (CS) and clustering have been prove to be proficient ways to reduce the energy consumptions in WSNs The idea is to separation of WSN into clusters, in which every cluster head collects the sensor readings within its cluster and forms CS measurements to be forwarded to the base station. In our Compressive Sensingbased clustering algorithm, a clustered WSN only wants to send $\mathrm{M}$ measurements from its clusters to the BS. All raw reading data from $\mathrm{N}$ sensors will be recovered based on those measurements at the BS. The algorithm helps to reduce a significant energy consumption to transmit data from the network to the BS. S. Mathapti et al. (2012), [4] have developed a new energy efficient routing protocol called energy efficient reliable routing protocol for WSN by using data aggregation technique. The main aim if data aggregation technique is to eliminate the redundant data transmission. In this paper we form clusters and coordinator nodes. The $\mathrm{CN}$ selects a cluster head $(\mathrm{CH})$ in each cluster based upon the energy level and the distance to the $\mathrm{CN}$. The packets sent by the sensor nodes are aggregated at the $\mathrm{CH}$ and transmitted to the $\mathrm{CN}$. The $\mathrm{CN}$ measures the loss ratio and compares it with a threshold value of loss ratio. Depending upon this value, the forward node count is incremented or decremented and the cluster size is adaptively changed, ensuring reliability and balanced energy consumption. Jeon et al. (2012), [5] have proposed high event density tree based cluster centered at the high even density area. The main idea behind this is to develop a multi hop short distance routing path for data transmission. We also develop the data gathering and aggregation sensor to realize HEDACR. The result of experiment indicates that HEDACR reduces the energy consumption and extends the network lifetime as compared with LEACH using one-hop. Alemu et al. (2012), [6] have discussed about the data aggregation framework for the wireless sensor network based upon a hierarchical multiparent cluster. To minimize the energy consumption is the major issue in the wireless sensor network. To resolve this issue efficient fault tolerance data aggregation framework is introduced based on hierarchical multi-parent clustering. It addresses how to verify faulty nodes, how to improve corrupted data and how to minimize energy and bandwidth for longer network lifetime. In which for aggregate sensed data cluster have two heads primary and secondary. Kim et al. (2010), [7] have discussed the TBC (tree based clustering) approach for energy efficient wireless sensor network.TBC is a cluster based protocol, several clusters are formed in TBC and each cluster has a cluster head $(\mathrm{CH})$. The nodes within the cluster construct a routing tree and the cluster head is the root of it. Cluster heads measure the distance between the member nodes and itself for the tree configuration process. In TBC every cluster is divided into levels. The height of the tree is determined based on the distance of the member nodes from the cluster head. TBC reduces and balances the energy consumptions among the nodes. Thus it extends the network lifetime as compared to other schemes. Xibei et al. (2010), [8] have discussed an improved protocol DCDA-LEACH. It makes improvement to LEACH with data correlation and data aggregation. It divides the nodes into regions. Each region is defined as atomic region, in which clusters are formed to reduce the energy consumption. In their paper they used multi-skip routing to decrease the number of cluster heads which communicate with base station directly. Therefore this protocol can balance the node energy and prolong the network lifetime. Lindsey et al. (2002), [9] have presented the protocol PEGASIS. PEGASIS is a chain based protocol. Firstly in PEGASIS information is gathered and transmit it base station via sensor nodes. The main idea behind PEGASIS to form a chain of sensor nodes. Each node can communicates with their neighbor node and transmit the gathered data node to node and then takes turn transmitting the data to the base station. PEGASIS does not include rounds procedure thus the amount of energy spent per round will be reduced. So PEGASIS performs better than the LEACH. PEGASIS shows the better results. Heinzelman et al. (2002), [10] have discussed about the protocol LEACH, In this paper he introduced a protocol architecture for micro sensor network that combine the energy proficient cluster based routing and media access mutually with particular data aggregation for increasing the lifetime system performance, latency and quality. For increasing the network lifetime LEACH include a distributed cluster formation technique, algorithms for adapt clusters and rotate cluster head position to equally allocate the energy load between all nodes.

\section{PROPOSED METHODOLOGY}

\section{/*starting of WSN*/}

1. 100 nodes are deployed randomly. /*Setup phase*/

2. $P_{S}(\mathrm{t})=\left\{\begin{array}{c}\frac{k}{N-k *\left(r \bmod \frac{N}{k}\right)}: H_{S}(t)=1 / / \text { selection of cluster head } \\ 0: H_{S}(t)=0\end{array}\right.$ Cluster head $(\mathrm{CH})$ selection criteria where $\mathrm{N}$ is the number of nodes, $\mathrm{k}$ is the number of clusters, $\mathrm{r}$ is the number of rounds, If $H_{S}(t)=1$, then nodes has not been a CLUSTER HEAD in the recent $\mathrm{r} \bmod (\mathrm{N} / \mathrm{k})$ rounds. And if $H_{s}(t)=0$, then nodes have been a CLUSTER HEAD.

3. Selected $\mathrm{CH}$ transmit the message to sensor nodes(SNs).

4. $\mathrm{CH}$ assign TDMA schedule to $\mathrm{CMs}$ (cluster members).

/*Transmission process*/

5.for $\mathrm{CH}$, for $\mathrm{CMs}$

6.Data transmit from $\mathrm{CMs}$ to $\mathrm{CH}$ according to TDMA schedule.

7.Intracluster Data aggregation by using existing data aggregation functions:

(a) Addition $=\sum_{i=1}^{X}\left(A_{i}\right)$ for $\forall\left(A_{i}\right)=$ Distinct Data $/ / i=1 \ldots X$, 
$\mathrm{X}=$ nodes having dissimilar data packets.

(b) Division $=\frac{1}{Y} \sum_{j=1}^{Y}\left(B_{j}\right)$ for $\forall\left(B_{J}\right)=$ Similar data

$\mathrm{j}=1 \ldots \mathrm{Y}, \mathrm{Y}=$ nodes having alike data packets.

8.end for $\mathrm{CMs}$

$/ *$ selection of pass on node $* /$

9. for pass on node

10. while status $=0 / /$ node is not working

11. find distance between current ${ }_{\mathrm{CH}}$ and Base Station using Euclidean distance:

distance $=\sqrt{\left(\mathrm{x}_{1}-\mathrm{x}_{2}\right)^{2}+\left(\mathrm{y}_{1}-\mathrm{y}_{2}\right)^{2}}$

12. if distance $<$ min_distance then

13. Assign distance to minidistance

14. Assign current $\mathrm{CH}_{\mathrm{CH}}$ to pass on node

15. end if

16. end while

17. Set status $=1 / /$ node is in working

/*Transmission process $2 * /$

18. Send data from $\mathrm{CHs}$ to pass on node.

19. End for CLUSTER HEAD

20. Intercluster Data Aggregation by using Additive Data aggregation function:

i. $\forall \mathrm{j} \in \mathrm{CH} / /$ for each node belong to cluster head

ii. if $j$. status = 'Inactive' // check the active or inactive status where active means it has been added for data aggregation previously and inactive means it require data aggregation

iii. if(maxidata $)=\emptyset$ then $/ /($ maxidata $)$ is a type of frame which stores the aggregated data.

iv. Assign j. data to maxidata

v. Set j. status = 'Active' // not necessary to aggregate data of $\mathrm{j}$ over again.

vi. else if maxidata $+\mathrm{j}$. data $\leq$ channel_limitthen //channel_limit is the maximum capacity of transferring data of Wireless sensor network.

vii. Assign maxidata $+\mathrm{j}$. data to maxidata

viii. $\quad$ Set j. status $=$ 'Active'

ix. else maxidata + j.data $\leq$ channel_limit then

x. Assign maxidatatoadditive_packet(m) additive_packet $(\mathrm{m})$ is a entire label.

xi. Put (maxidata) $=\varnothing$

xii. $m=m+1 / /$ start a new additive packet

xiii. Repeat steps (iii)to (xii)till the status of all CLUSTER HEADs not equal to inactive.

xiv.en, end

/* reduce data by using LZW Compression technique*/

21. $\mathrm{CPS}=\frac{\mathrm{APS}}{0.35} / / \mathrm{CPS}$ is Compressed packets size, APS is real packet size, Maximum compression ratio of $\mathrm{LZW}=0.35$

/*Transmission phase $3 * /$

22. Send compressed data from pass on node to BS.

23. End for pass on node

24. Extract data by using LZW decompression at BS

25. Apply divisible data aggregation function at base station

i. Repeat step 25

ii. While the count_additive_packet not equal to ZERO

iii. Extract data from packet(count_additive_packet) and Set count_additive_packet

$$
=\text { count_additive_paket }-1 \text {. }
$$

iv. Finish
26. End of code

\section{RESULTS AND DISCUSSION}

In this approach, the network is divided into various clusters. A cluster is formed by sensor nodes and then selects the cluster heads from the clusters. Cluster heads will selected on the bases of probability function .The sensor nodes transmit the collected data to the cluster head, then cluster head send the collected data to the root cluster head where all compressed data is stored, and then data is sent to the base station. Clustering helps in reducing power consumption and collisions. In this research work, clustering and compressed data is considered. data will be compressed by using compressive sensing technique. sensing based. If the sensor nodes present in the network have the equal amount of energy, then the network is called homogeneous sensor network.

Table 1 has shown the various parameters required to successfully simulate the WSNs. The taken values are benchmark for WSNs.

Table 1: WSNs characteristics

\begin{tabular}{|c|c|}
\hline Parameter & Value \\
\hline Area(x,y) & 100,100 \\
\hline Base station(x,y) & 50,150 \\
\hline Nodes(n) & 100 \\
\hline Probability(p) & 0.1 \\
\hline Initial Energy & $0.1 \mathrm{~J}$ \\
\hline transmiter_energy & $50 * 10^{-9} \mathrm{~J} / \mathrm{bit}$ \\
\hline receiver_energy & $50 * 10^{-9} \mathrm{~J} / \mathrm{bit}$ \\
\hline Free space(amplifier) & $10 * 10^{-13} \mathrm{~J} / \mathrm{bit} / \mathrm{m}^{2}$ \\
\hline Multipath(amplifier) & $0.0013 * 10^{-13}$ \\
& $\mathrm{~J} / \mathrm{bit} / \mathrm{m}^{2}$ \\
\hline Effective Data aggregation & $5 * 10^{-9} \mathrm{~J} / \mathrm{bit} / \mathrm{signal}$ \\
\hline Maximum lifetime & 4000 \\
\hline Data packet Size & $4000 \mathrm{~KB}$ \\
\hline m (fraction of advanced nodes) & 0.2 \\
\hline $\begin{array}{c}\text { a (energy factor between normal } \\
\text { and advanced nodes) }\end{array}$ & 1 \\
\hline LZW compression & 0.35 \\
\hline
\end{tabular}

\subsection{Performance Evaluation}

FIRST NODE DEAD: - Table 2 shows the first node dead evaluation of the GSTEB and the improved GSTEB protocols. In the table, it is clearly shown that the improved GSTEB performs better as compared to the GSTEB.

Table 2: First Node Dead Evaluation

\begin{tabular}{|c|c|c|}
\hline Energy level & GSTEB & Improved GSTEB \\
\hline 0.01 & 18 & 40 \\
\hline 0.02 & 36 & 101 \\
\hline 0.03 & 56 & 147 \\
\hline
\end{tabular}




\begin{tabular}{|l|l|l|}
\hline 0.04 & 72 & 186 \\
\hline 0.05 & 90 & 251 \\
\hline 0.06 & 108 & 251 \\
\hline 0.07 & 144 & 294 \\
\hline 0.08 & 167 & 426 \\
\hline 0.09 & 189 & 475 \\
\hline 0.1 & 224 & 533 \\
\hline
\end{tabular}

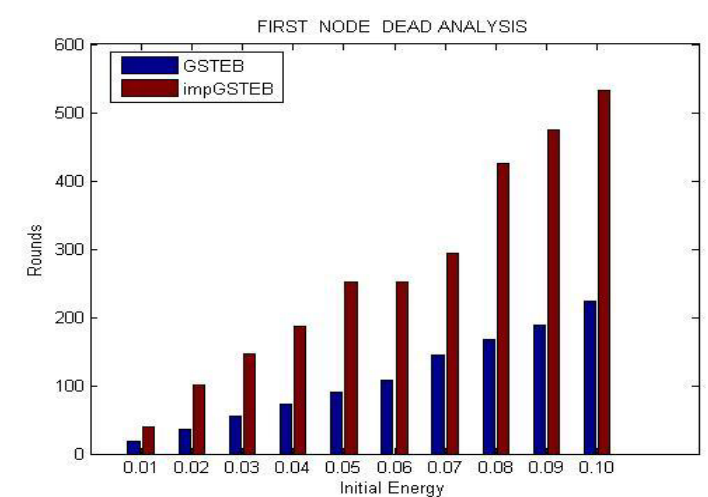

Fig 2: First Node Dead Analysis

Fig. 2 is showing the comparison of GSTEB and the improved GSTEB with respect to total number of rounds in case of first dead node. $\mathrm{X}$-axis is representing initial energy. $\mathrm{Y}$-axis is representing the number of rounds. It has been clearly shown that the overall number of rounds in case of improved GSTEB is quite more than that of the GSTEB. Thus improved GSTEB outperforms over the GSTEB.

TENTH NODE DEAD:-Table 3 shows the tenth node dead evaluation of the GSTEB and the improved GSTEB protocols. In the table, it is clearly shown that the improved GSTEB performs better as compared to the GSTEB.

Table 3: Tenth Node Dead Evaluation

\begin{tabular}{|c|c|c|}
\hline Energy level & GSTEB & $\begin{array}{c}\text { Improved } \\
\text { GSTEB }\end{array}$ \\
\hline 0.01 & 22 & 52 \\
\hline 0.02 & 44 & 112 \\
\hline 0.03 & 60 & 170 \\
\hline 0.04 & 85 & 280 \\
\hline 0.05 & 102 & 341 \\
\hline 0.06 & 121 & 396 \\
\hline 0.07 & 159 & 462 \\
\hline 0.08 & 182 & 507 \\
\hline 0.09 & 211 & 586 \\
\hline 0.1 & 248 & \\
\hline
\end{tabular}

Fig. 3 is showing the comparison of GSTEB and the improved GSTEB with respect to total number of rounds in case of tenth dead node. $\mathrm{X}$-axis is representing initial energy. $\mathrm{Y}$-axis is representing the number of rounds. It has been clearly shown that the overall number of rounds in case of improved GSTEB is quite more than that of the GSTEB. Thus improved GSTEB outperforms over the GSTEB.

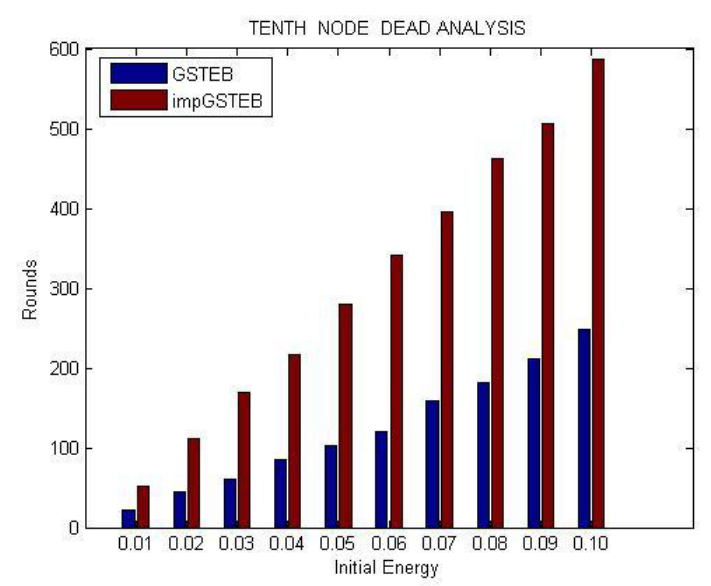

Fig 3: Tenth Node Dead Analysis

LAST NODE DEAD: - Table 4 shows the last node dead evaluation of the GSTEB and the improved GSTEB protocols. In the table, it is clearly shown that the improved GSTEB performs better as compared to the GSTEB.

Table 4: Last Node Dead Evaluation

\begin{tabular}{|c|c|c|}
\hline Energy level & GSTEB & $\begin{array}{c}\text { Improved } \\
\text { GSTEB }\end{array}$ \\
\hline 0.01 & 50 & 82 \\
\hline 0.02 & 100 & 168 \\
\hline 0.03 & 150 & 234 \\
\hline 0.04 & 200 & 311 \\
\hline 0.05 & 250 & 383 \\
\hline 0.06 & 300 & 458 \\
\hline 0.07 & 350 & 528 \\
\hline 0.08 & 400 & 586 \\
\hline 0.09 & 450 & 664 \\
\hline 0.1 & 500 & 753 \\
\hline
\end{tabular}

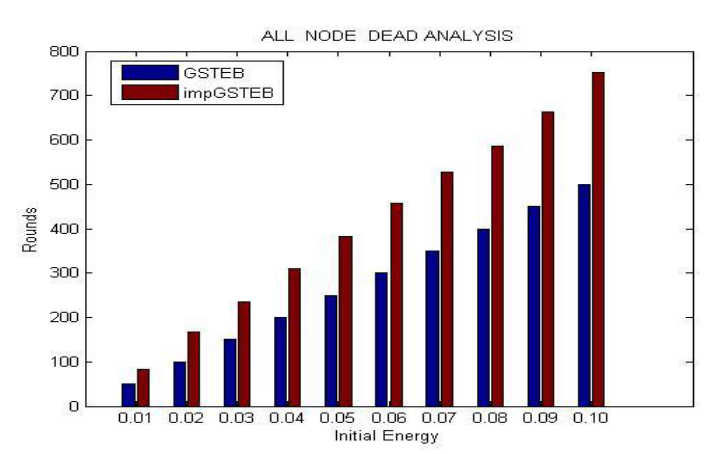

Fig 4: Last Node Dead Analysis 
Fig.4 is showing the comparison of GSTEB and the improved GSTEB with respect to total number of rounds in case of last dead node. $\mathrm{X}$-axis is representing initial energy. $\mathrm{Y}$-axis is representing the number of rounds. It has been clearly shown that the overall number of rounds in case of improved GSTEB is quite more than that of the GSTEB. Thus improved GSTEB outperforms over the GSTEB.

\section{CONCLUSION}

In this paper, an enhancement in the tree-based protocol has been done by using clustering and compressive sensing techniques for wireless sensor networks. The proposed technique has been designed and implemented in the MATLAB tool. From the survey, it has been found that though many protocols has been proposed so far to improve the energy efficiency further but still much enhancement could be done which has done in this paper. Moreover a comparison has been done of the proposed and the existing technique. From the comparison, it has been concluded that the proposed technique gives efficient results in terms of stable period and network lifetime. Further we can add security to our WSN by encrypting the data using artificial intelligence concept.

\section{REFERENCES}

[1] Han, Z. Wu, J. Liu, L. and Tian, K. 2014. A General Self Organized Energy Balancing Routing Protocol for Wireless Sensor Network, IEEE Transaction on Nuclear Science, VOL 61, No. 2, pp 732-740

[2] Hwang, S. Park, J. and Kim,D. 2013. School of Electrical \& Electronic Engineering Yonsei University Seoul, South Korea main technical program at IFIP WMNC.

[3] Minh Tuan Nguyen and Nazanin Rahnavard School of Electrical and Computer Engineering Oklahoma State
University Stillwater, OK 740782013 IEEE Military Communications Conference.

[4] Mathapati, Basavaraj, S. Patil, S.R. and Mytri, V.D. 2012. Energy Efficient Reliable Data Aggregation Technique for Wireless Sensor Networks, In Computing Sciences (ICCS), 2012 International Conference on, pp. 153-158.

[5] Jeon, Boungju, Kang, B. and Park, S. 2012.Design and implementation of high event density area centered clustering based routing, In Computing, Networking and Communications (ICNC), 2012 International Conference on, pp. 247-251.

[6] Alemu, Yihun, Koh, J. B. and Kim, D.K. 2010. A Hierarchical Multi-Parent Cluster-Based Data Aggregation Framework for WSNs, In Complex, Intelligent and Software Intensive Systems (CISIS), 2010 International Conference on, pp. 549-554.

[7] Kim, K.T. and Youn, H.Y. 2010. Tree-Based Clustering (TBC) for energy efficient wireless sensor networks, in Proc. AINA 2010, pp. 680-685.

[8] Xibei, Jia, Huazhong, Z. and Jingchen, Z. 2010. Research of data aggregation routing protocol in WSN data-related applications, In Computer Science and Information Technology (ICCSIT), pp. 647-651.

[9] Lindsey, S. and Raghavendra, C. 2002. Pegasis: Powerefficient gathering in sensor information systems, in Proc. IEEE Aerospace Conf., pp. 1125-1130.

[10] Heinzelman, W.R., Chandrakasan, A. and Balakrishnan, H. 2000. Energyefficient communication protocols for wireless micro sensor networks, in Proc. 33rd Hawaii Int. Conf. System Sci., pp. 3005-3014. 